\title{
ON TIME SERIES MODEL ORDER SELECTION OF NON- STATIONARY AND NON-NORMAL DATA STRUCTURE
}

\author{
H. A. Chamalwa ${ }^{a^{*}}$, H. R. Bakarib and I. Akeyede ${ }^{c}$ \\ ${ }^{a, b}$ Departmemnt of Mathematical Sciences, University of Maiduguri \\ ${ }^{c}$ Department of Statistics, Federal University Lafia, PMB 146, Lafia Nigeria \\ *Corresponding Author: chamalwa@gmail.com,
}

\begin{abstract}
The study is aimed at identifying the orders of Time Series Models in Non-Stationarity Non-normal data structure from Uniform distributions with a view to determining the best Autoregressive/ Moving Average orders from time series models (ARMA and ARIMA) under different underline distributions when Non-Stationarity assumption is assumed. The data is generated from Autoregressive (AR) linear of second orders of general classes of Autoregressive functions. The generation of the data used for this simulation study is non-stationary cases and nonnormal. The data were simulated for both response variables and error terms from non-normal Distribution. The result shows that the values of the penalty function: AIC, BIC, HQIC and FPE of the order selection increases with increase in sample size but decreases with increasing order. It was observed that at both lower $(20,40)$ and larger $(160,180$ and 200$)$ sample sizes, models with smallest orders. Similarly, the selection process is tied to the principle of parsimony i.e smaller orders are selected, but vary with the variation in the distribution of the series. The study recommends the need to develop a methodology for model selection combining objective and subjective techniques.
\end{abstract}

Keywords: Data, Generation, Linear, Normal, Results, Simulation

\section{0: Introduction}

Time series analysis in its entirety serves two broad purposes which are, to model a successive observation of a given variable as well as to provide a better understanding of the stochastic mechanism that characterized any observed series and secondly to make predictions of the future values of the observed series on the basis of the historical observation and other possibly relevant factors. Therefore, the need to have a proper and better description of the series cannot be over emphasis. Since, the primary objective of the model selection process is to assess competing models and select the best that describes the data Ongbali, Igboanugo, Afolalu, Udo and Okokpujie (2018). Modeling and forecasting are the main stay of any time series analysis or investigation; in modeling aspect, order identification is very crucial, as the parameters of the fitted model depends largely on the correct order selected and subsequent estimation and performance, as there should be method that is suitable for fitting particular model. Model identification is still a 'thorny issue' in robust time series analysis (Martin and Yohai 1986). As Chang (1982) remarks: 'We need to protect not only the parameter estimation process against the adverse effect of exogenous interventions but also the model identification process so that appropriate model forms for the underlying time series can be specified in the very first place.' Knowledge of the data generating mechanism also plays a great role in the identification of the 
Hamidu Aliyu Chamalwa / International Journal of Research Publications (IJRP.ORG)

tentative model (Cryer and Chan, 2008). Hastie, Tibshirani and Friedman (2009) opined that cardinal rise reason 2967 model identification is to assess the performance of different models and select the best fit among the several models for particular data. the use final prediction Error (FPE) for model order selection being the expected variance of prediction error when an Autoregressive(AR) model was fitted was suggested and objective of model selection include finding a good predictor that describes a system and Akaike Information Criterion (AIC) is a principal model selection method (Burnham, Anderson and Huyvaert , 2011)

Numerous literatures (Beguin, 1980, Davies 1984, Tsay and Tiao 1985, Choi, 1992, Chan, 1999, Eija, 2015, Norhayati, 2016) abound on order selection since the pioneer work of Box and Jenkins in (1975) but not yet extensive. All of them failed to consider different distributions of error terms and responses in respect to both stationary and non-stationary data structures. More so, the ACF and PACF frequently used in the literature for identification of models' orders depend on the size of the series and very sensitive to outliers or violation of the ideal normal assumption (Stadnytskaet'al, 2008). This study therefore intends to identify a suitable order for the different time series models under different distributions and sample size in fitting and forecasting stationary and non-stationary data structures using different criteria.

\subsection{Methodology}

For non-stationary cases, the data will be simulated for response variables from Uniform distribution with the parametres of 200, 32 and 38 while that of error terms from normal distribution with mean 1000 and variance 10, so as to violate the white noise assumption of zero mean and difference means and variances of the responses and error terms, thereby violate the Stationarity assumptions i.e;

$$
Y_{t i} \sim N(200,20) \text { ande }_{t i} \sim N(200,10) \text { For non Stationarity cases }
$$

$t=1,2, \ldots, 20,40,60,80,100,120,140,160,180$ and $200 . i=1,2, \ldots, 1000$

The generation of the data used for this simulation study is non-stationary cases.

$\mathrm{x}<\mathrm{w}-\operatorname{runif}(120,32,38)$

$\mathrm{x}[\mathrm{t}]<-0.7 * \mathrm{x}[\mathrm{t}-1]+0.9 * \mathrm{x}[\mathrm{t}-2]+\mathrm{w}[\mathrm{t}]$

A Non stationary data from a Non-Normal data structures were simulated at various sample sizes of 20, 40, 60, 80, 100, 120, 140, 160, 180 and 200 respectively. Several ARMA (p, q) were fitted on the simulated data; the four different models considered are: ARMA $(1,1)$, ARMA $(1,2), \operatorname{ARMA}(2,1)$ and ARMA $(2,2), \operatorname{ARIMA}(1,1,1), \operatorname{ARIMA}(1,1,2), \operatorname{ARIMA}(2$, $1,1)$ and ARIMA $(2,1,2)$ respectively.

The effect of different levels of ORDER $(0.3,0.6$, and -0.3, -0.6) at the sample size of 20,40,60, 80, 100, 120, 140, 160, 180 and 200 which represent small, moderate and large sample sizes respectively on the simulated data from the non-stationary and non-normal data . The simulation study was carried out with 1000 iteration on each case in R Statistical software. respectively.

\section{1: Model Assessment Criteria}


The goodness of fit for each model was assessed using the criteria: of AIC, BIC, HQIC and FPE. The model with hit lowe value is considered the best among the models for the simulated data. Penalty function are equally widely used for model identification: Akaike Information Criterion (AIC), Bayesian Information Criterion (BIC) and Hannan-Quin Information Criterion (HQIC) and Final Prediction Error (FPE)

$$
\begin{aligned}
& \mathrm{AIC}=\log \left[\left(\hat{\sigma}_{p, q}^{2}\right)+\frac{(p+q) 2}{n}\right. \\
& \mathrm{BIC}=\log \left[\left(\hat{\sigma}_{p, q}^{2}\right)+\frac{(p+q) \log (n)}{n}\right. \\
& \mathrm{HQIC}=\log \left[\left(\hat{\sigma}_{p, q}^{2}\right)+\frac{(p+q) 2 \log (\log (n))}{n}\right. \\
& \mathrm{FPE}(\mathrm{p})=\sigma_{p}^{2}\left(1+\frac{p}{N}\right), \text { where }_{p}^{2}=\frac{N}{N-p} \hat{\sigma}^{2} \\
& \mathrm{FPE}(\mathrm{p})=\frac{N}{N-p}\left(1+\frac{p}{N}\right) \hat{\sigma}^{2}=\frac{N+p}{N-p} \hat{\sigma}_{p}^{2}
\end{aligned}
$$

\section{Analysis, Results and Discussion}

The orders of each model family considered are investigated and the results were presented in tables according to the model families at various sample sizes of $20,40,60,80,100,120,140,160,180$ and 200 respectively and the criteria for the assessment, at different sample sizes. Here, the data were simulated from normal of both observed values and error terms to achieve the normality assumption. The criteria like AIC, BIC, HQIC and FPE are used to determine the models' orders such that a model's order with the least criteria is chosen for the model's family.

\section{1: Order Determination for ARMA on Stationary Data Structure from Non-Normal Distribution}

From each iteration simulated, the values of the criteria for the assessment (AIC and BIC) were computed and their average values were recorded according to sample sizes as shown in table 3.1. The values from the tables were plotted in figures 3.1a and $3.1 \mathrm{~b}$ respectively. The model with lowest criteria value is considered as the best. For each of the iteration the values of the criteria for the assessment (AIC, BIC, HQIC and FPE) were computed and their average values were recorded according to

\begin{tabular}{|c|c|c|c|c|c|c|c|c|}
\hline $\begin{array}{l}\text { Sample } \\
\text { Sizes }\end{array}$ & $\begin{array}{l}\text { ARMA( } \\
\mathbf{1 , 1})\end{array}$ & $\begin{array}{l}\text { ARMA } \\
(1,2)\end{array}$ & $\begin{array}{l}\text { ARMA } \\
(2,1)\end{array}$ & $\begin{array}{l}\text { ARMA } \\
(2,2)\end{array}$ & $\begin{array}{l}\text { ARMA } \\
(1,1)\end{array}$ & $\begin{array}{l}\text { ARMA } \\
(1,2)\end{array}$ & $\begin{array}{l}\text { ARMA } \\
(2,1)\end{array}$ & $\begin{array}{l}\text { ARMA } \\
(2,2)\end{array}$ \\
\hline 20 & 110.295 & 115.601 & 112.926 & 105.838 & 114.2785 & 120.5797 & 117.905 & 111.8131 \\
\hline 40 & 194.483 & 187.526 & 190.616 & 201.834 & 201.2392 & 195.9707 & 199.061 & 211.9681 \\
\hline 60 & 282.374 & 285.84 & 280.839 & 280.850 & 290.7514 & 296.3118 & 291.310 & 292.7765 \\
\hline
\end{tabular}
sample sizes as shown in tables above

Table 3.1: AIC and BIC Values of ARMA (p) Model for UNIFORM Data
AIC
BIC 


\begin{tabular}{|c|c|c|c|c|c|c|c|c|}
\hline \multicolumn{9}{|c|}{ Hamidu Aliyu Chamalwa / International Journal of Research Publications (IJRP.ORG) } \\
\hline 80 & 368.496 & 346.248 & 390.522 & 370.153 & 378.0245 & 358.1585 & 402.432 & 384.4456 \\
\hline 100 & 453.962 & 443.504 & 452.556 & 454.149 & 464.383 & 456.5299 & 465.581 & 469.7808 \\
\hline 120 & 447.750 & 582.033 & 524.198 & 541.950 & 458.1709 & 595.9712 & 538.136 & 558.6753 \\
\hline 140 & 638.439 & 604.282 & 592.576 & 590.849 & 650.2057 & 618.9902 & 607.284 & 605.4997 \\
\hline 160 & 738.885 & 672.108 & 681.703 & 670.410 & 751.1865 & 687.4843 & 697.079 & 688.8611 \\
\hline 180 & 764.501 & 766.719 & 777.199 & 775.848 & 777.2734 & 782.6839 & 793.164 & 795.0064 \\
\hline 200 & 912.324 & 851.023 & 846.967 & 841.241 & 925.5177 & 867.515 & 863.458 & 861.0312 \\
\hline
\end{tabular}

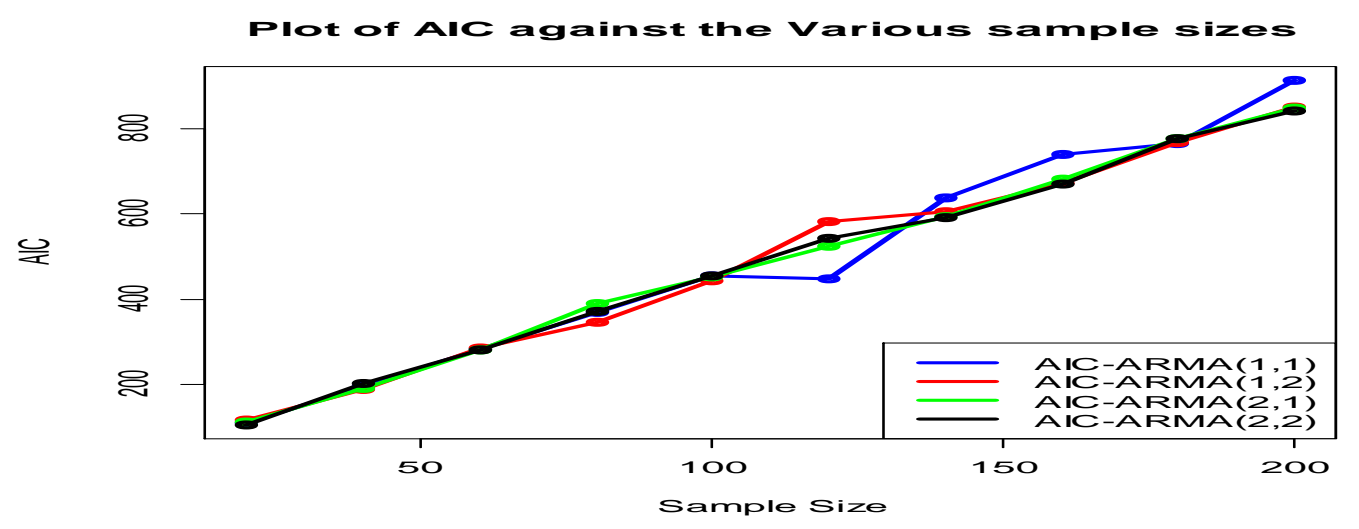

Fig.3.1a: AIC values for ARIMA (p, d, q) Models form a Non-normal Data

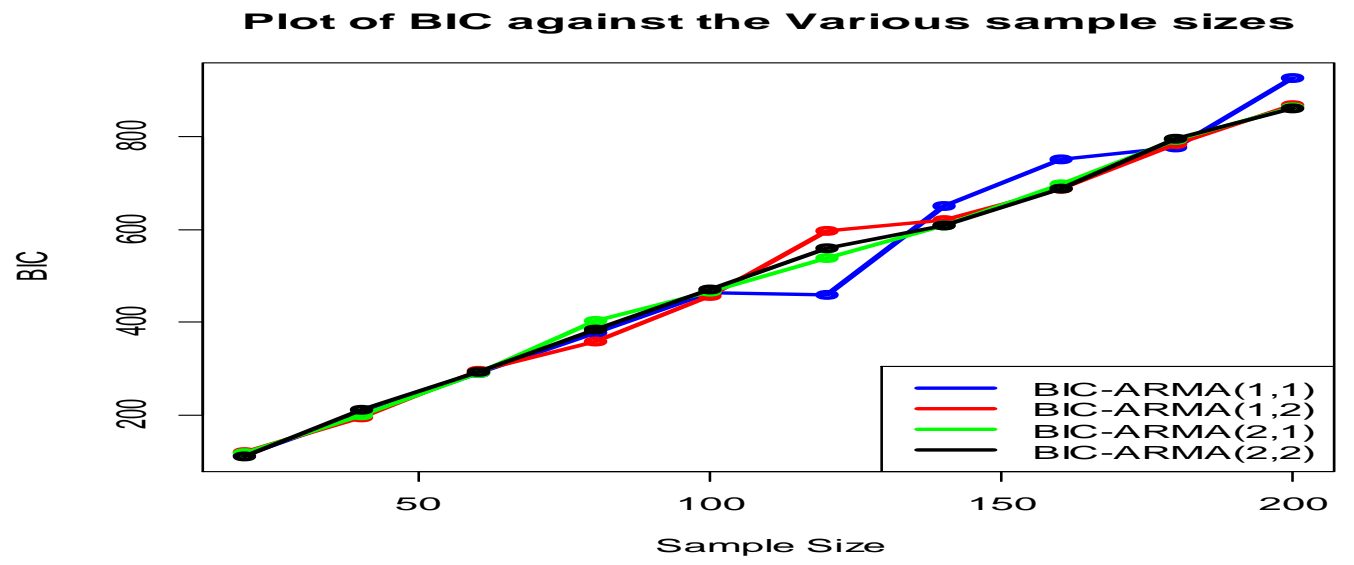

Fig.3.1b: BIC values for ARIMA (p, d, q) Models form a Non-normal Data 


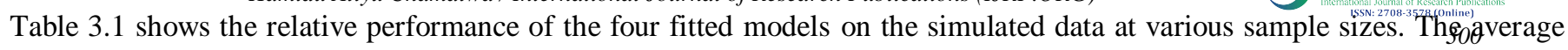
values of AIC and BIC for each of the models are recorded in the table above and then plotted in figures $3.1 \mathrm{a}$ and $3.1 \mathrm{~b}$ respectively. At sample sizes 20,140,160 and 200 ARMA $(2,2)$ was selected as the best fit. ARMA $(2,1)$ was the best fit at sample sizes 60 and 120. While $\operatorname{ARMA}(1,2)$ was the best fit at sample sizes 40, 80 and 100 respectively.

Table 3.2: HQIC and FPE Values of ARMA (p) Model for Stationary Non-normal Data( UNIFORM )

HQIC FPE

\begin{tabular}{|c|c|c|c|c|c|c|c|c|}
\hline $\begin{array}{l}\text { Sample } \\
\text { Sizes }\end{array}$ & $\begin{array}{l}\text { ARMA( } \\
\mathbf{1 , 1})\end{array}$ & $\begin{array}{l}\text { ARMA } \\
(\mathbf{1}, \mathbf{2})\end{array}$ & $\begin{array}{l}\text { ARMA } \\
(2,1)\end{array}$ & $\begin{array}{l}\text { ARMA } \\
(2,2)\end{array}$ & $\begin{array}{l}\text { ARMA } \\
(\mathbf{1}, \mathbf{1})\end{array}$ & $\begin{array}{l}\text { ARMA } \\
(\mathbf{1}, 2)\end{array}$ & $\begin{array}{l}\text { ARMA } \\
(2,1)\end{array}$ & $\begin{array}{l}\text { ARMA } \\
(2,2)\end{array}$ \\
\hline 20 & 107.143 & 115.717 & 107.077 & 106.511 & 68.02588 & 72.34176 & 66.4970 & 64.63 \\
\hline 40 & 197.331 & 190.742 & 192.882 & 194.093 & 110.1149 & 104.7689 & 106.012 & 105.1989 \\
\hline 60 & 281.677 & 271.916 & 270.576 & 293.316 & 150.99 & 144.0379 & 143.297 & 154.3058 \\
\hline 80 & 385.265 & 355.420 & 380.140 & 361.475 & 202.8649 & 185.187 & 198.510 & 186.8578 \\
\hline 100 & 463.163 & 424.137 & 441.477 & 452.271 & 241.0412 & 218.6998 & 227.906 & 232.0155 \\
\hline 120 & 514.856 & 513.568 & 528.608 & 528.600 & 265.6905 & 263.3672 & 271.272 & 269.6223 \\
\hline 140 & 614.786 & 595.521 & 610.501 & 609.037 & 315.8526 & 304.1307 & 311.948 & 309.5167 \\
\hline 160 & 698.926 & 688.554 & 704.754 & 671.923 & 357.759 & 350.6888 & 359.098 & 340.3689 \\
\hline 180 & 796.383 & 771.138 & 822.278 & 787.773 & 406.5805 & 391.8268 & 418.263 & 398.7229 \\
\hline 200 & 924.504 & 850.939 & 870.839 & 865.413 & 471.1764 & 431.5553 & 441.808 & 437.295 \\
\hline
\end{tabular}


PIot of HQIC against the Various sample sizes

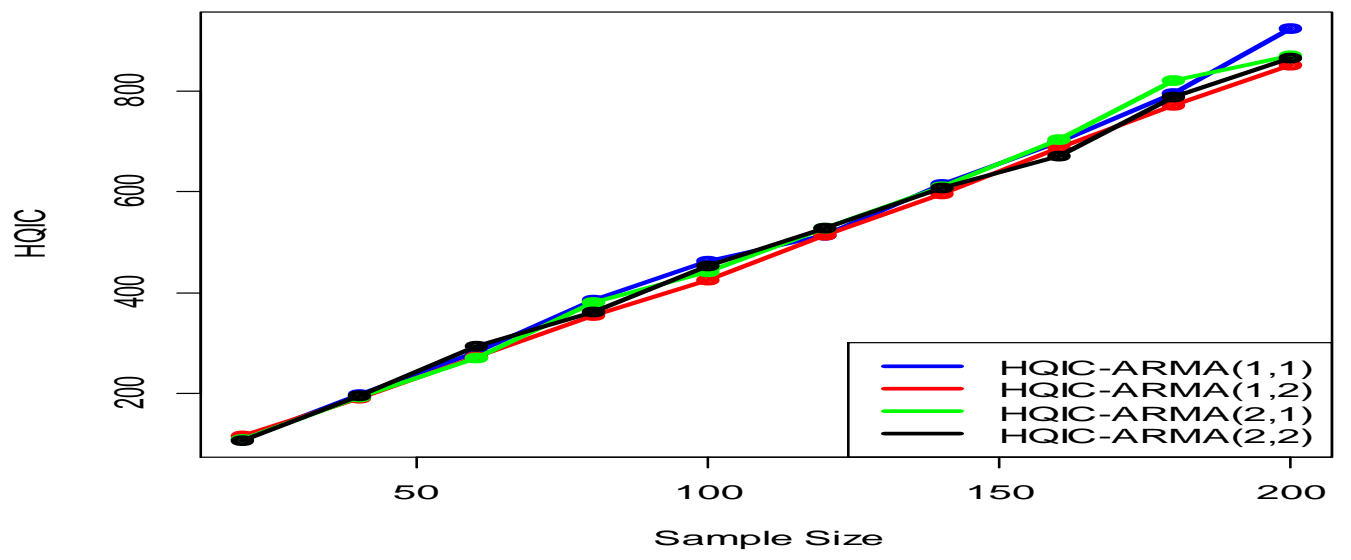

Fig.3.2a: AIC values for ARIMA (p, d, q) Models form a Non-normal Data

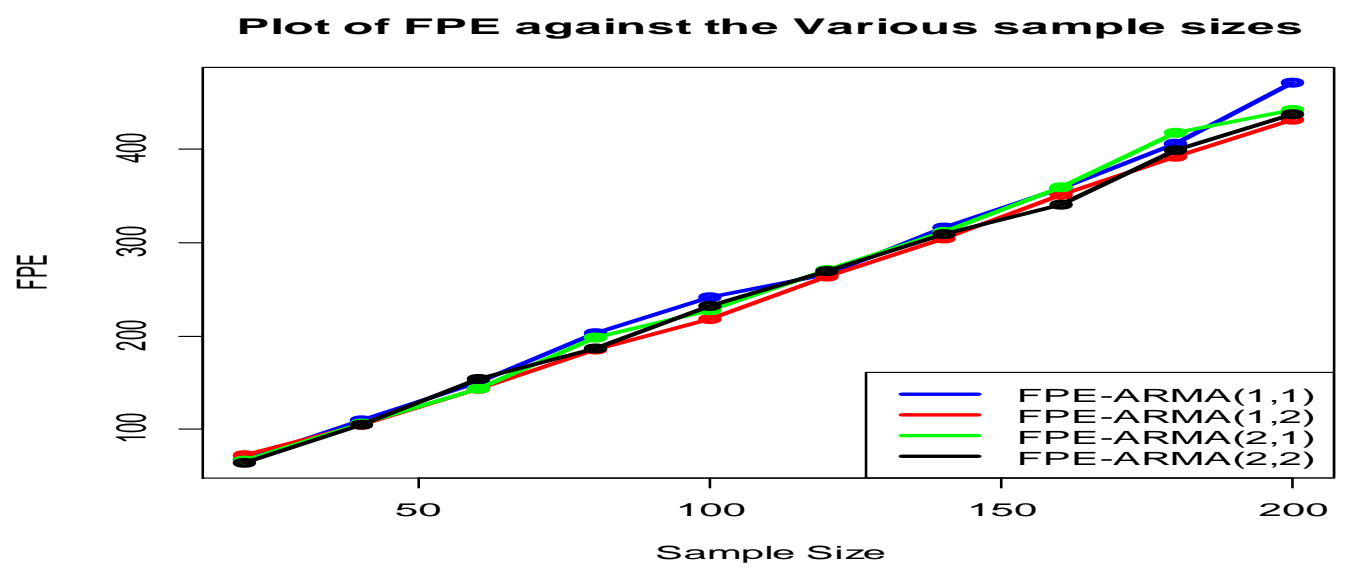

Fig.3.2b: AIC values for ARIMA (p, d, q) Models form a Non-normal Data

Similarly, table 3.2 above shows the relative performances of the four fitted models on the simulated data at various sample sizes are presented. The average values of HQIC and SIC for each of the models are recorded in the table above and plotted in figures 3.2a and 3.2b respectively. It was observed that the values of both AIC and BIC increases with increase in sample sizes of the simulated data. At sample sizes 20,140,160 and 200 ARMA $(2,2)$ was selected as the best fit. ARMA $(2,1)$ was the best fit at sample sizes 60 and 120. While ARMA $(1,2)$ was the best fit at sample sizes 40, 80 and 100 respectively.

\section{2: Determination of Best order of ARIMA Based on AIC and BIC Criteria}

From each iteration simulated, the values of the criteria for the assessment (AIC and BIC) were computed and their average values were recorded according to sample sizes as shown in table 3.3. The values from the tables were plotted in figures 3.3 and $3.3 \mathrm{~b}$ respectively. The model with lowest criteria value is considered as the best. 
AIC

BIC

\begin{tabular}{|c|c|c|c|c|c|c|c|c|}
\hline $\begin{array}{l}\text { Sample } \\
\text { Sizes }\end{array}$ & $\begin{array}{l}\text { ARIMA( } \\
1,1,1)\end{array}$ & $\begin{array}{l}\text { ARIMA } \\
(\mathbf{1 , 1 , 2 )}\end{array}$ & $\begin{array}{l}\text { ARIMA } \\
(2,1,1)\end{array}$ & $\begin{array}{l}\text { ARIMA } \\
(2,1,2)\end{array}$ & $\begin{array}{l}\text { ARIMA } \\
(\mathbf{1}, \mathbf{1 , 1})\end{array}$ & $\begin{array}{l}\text { ARIMA } \\
(1,1,2)\end{array}$ & $\begin{array}{l}\text { ARIMA } \\
(\mathbf{2}, \mathbf{1 , 1})\end{array}$ & $\begin{array}{l}\text { ARIMA } \\
(2,1,2)\end{array}$ \\
\hline 20 & 102.756 & 108.535 & 110.195 & 108.164 & 105.59 & 108.4151 & 113.973 & 112.887 \\
\hline 40 & 202.937 & 188.239 & 212.130 & 187.926 & 207.9285 & 194.8939 & 218.785 & 196.2447 \\
\hline 60 & 292.051 & 278.122 & 290.181 & 274.508 & 298.2836 & 286.4323 & 298.491 & 284.8965 \\
\hline 80 & 373.930 & 360.644 & 358.135 & 357.878 & 381.0387 & 370.1222 & 367.613 & 369.7259 \\
\hline 100 & 463.040 & 458.664 & 448.306 & 441.796 & 470.8255 & 469.0448 & 458.686 & 454.7721 \\
\hline 120 & 570.121 & 521.541 & 543.579 & 537.352 & 578.4584 & 532.6576 & 554.696 & 551.2484 \\
\hline 140 & 614.511 & 597.187 & 624.306 & 584.610 & 623.3148 & 608.9254 & 636.044 & 599.2827 \\
\hline 160 & 726.655 & 712.008 & 712.051 & 681.150 & 735.8625 & 724.2845 & 724.326 & 696.4951 \\
\hline 180 & 822.195 & 765.903 & 782.957 & 729.186 & 831.758 & 778.6526 & 795.707 & 745.1232 \\
\hline 200 & 898.535 & 850.923 & 861.888 & 853.599 & 908.4151 & 864.097 & 875.061 & 870.0656 \\
\hline
\end{tabular}


Plot of AIC against the Various sample sizes

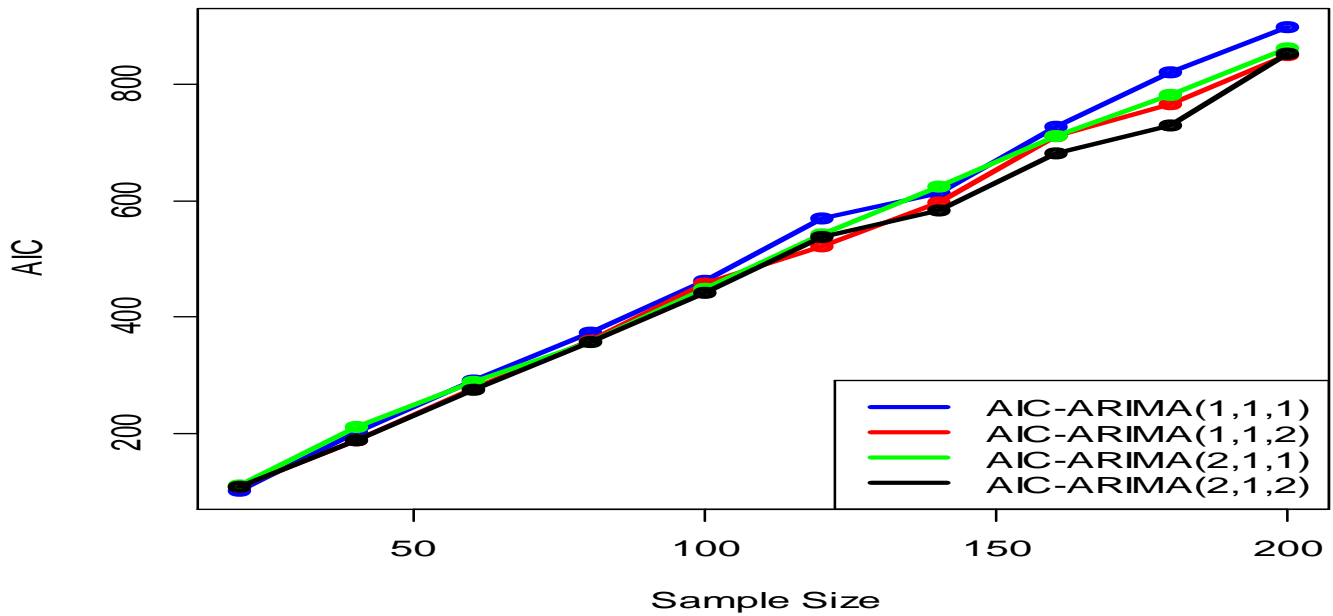

Fig.3.3a: AIC values for ARIMA (p, d, q) Models from a Non-normal Data

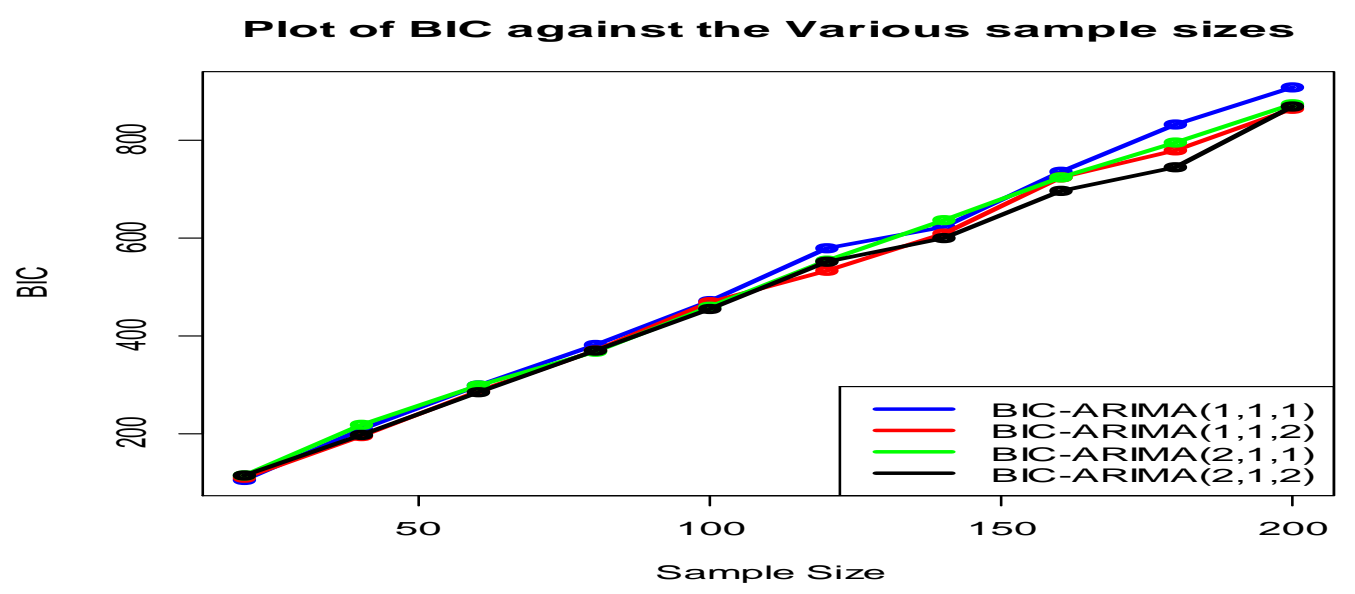

Fig.3.3b: BIC values for ARIMA (p, d, q) Models from a Non-normal Data

Table 3.3 above shows the relative performance of the four fitted models on the simulated data with 1000 iterations at various sample sizes. The average values of AIC and BIC for each of the model are recorded in the table above and then plotted in figures 3.3a and 3.3b respectively. At sample sizes 20, ARIMA $(1,1,1)$ was selected as the best fit. ARMA $(2,1,2)$ was the best fit at sample sizes 40, 60, 80,140, 160, 180 and 200 respectively. While ARIMA $(1,1,2)$ was the best fit at 120

Table 3.4 HQIC and FPE Values of ARIMA (p) Model for Stationary UNIFORM Data

HQIC

FPE

\begin{tabular}{lllllllll}
\hline Sample & ARIMA ( & ARIMA & ARIMA & ARIMA & ARIMA & ARIMA & ARIMA & ARIMA \\
Sizes & $\mathbf{1 , 1 , 1})$ & $\mathbf{( 1 , 1 , 2 )}$ & $\mathbf{( 2 , 1 , 1 )}$ & $\mathbf{( 2 , 1 , 2 )}$ & $\mathbf{( 1 , 1 , 1 )}$ & $\mathbf{( 1 , 1 , 2 )}$ & $\mathbf{( 2 , 1 , 1 )}$ & $\mathbf{( 2 , 1 , 2 )}$ \\
\hline $\mathbf{2 0}$ & 98.4687 & 102.163 & 109.063 & 92.5975 & 53.64235 & 64.65706 & 69.3247 & 56.70176
\end{tabular}




\begin{tabular}{|c|c|c|c|c|c|c|c|c|c|}
\hline \multirow[b]{2}{*}{40} & \multicolumn{7}{|c|}{ Hamidu Aliyu Chamalwa / International Journal of Research Publications (IJRP.ORG) } & \multicolumn{2}{|c|}{ ○) IJRP.ORG } \\
\hline & 196.121 & 192.831 & 192.431 & 190.682 & 126.1213 & 107.5 & 107.267 & $102.3827^{5 \mathrm{~N}}$ & 304 \\
\hline 60 & 267.178 & 277.857 & 287.237 & 273.896 & 144.5353 & 148.8789 & 154.062 & 145.1321 & \\
\hline 80 & 391.59 & 357.765 & 352.845 & 366.100 & 207.8665 & 188.0435 & 185.391 & 190.9431 & \\
\hline 100 & 478.188 & 452.143 & 455.803 & 463.097 & 250.6404 & 235.1904 & 237.133 & 239.3847 & \\
\hline 120 & 556.144 & 532.496 & 575.216 & 541.568 & 289.0395 & 274.9628 & 297.418 & 287.5723 & \\
\hline 140 & 640.450 & 616.526 & 616.186 & 620.641 & 330.9145 & 316.7607 & 316.583 & 317.2408 & \\
\hline 160 & 723.697 & 696.066 & 702.806 & 697.454 & 372.3045 & 356.2744 & 359.773 & 355.3089 & \\
\hline 180 & 806.649 & 767.843 & 772.523 & 780.898 & 413.5903 & 391.8268 & 394.246 & 396.8722 & \\
\hline 200 & 888.189 & 854.864 & 857.964 & 815.899 & 454.1842 & 435.2959 & 436.893 & 413.5017 & \\
\hline
\end{tabular}

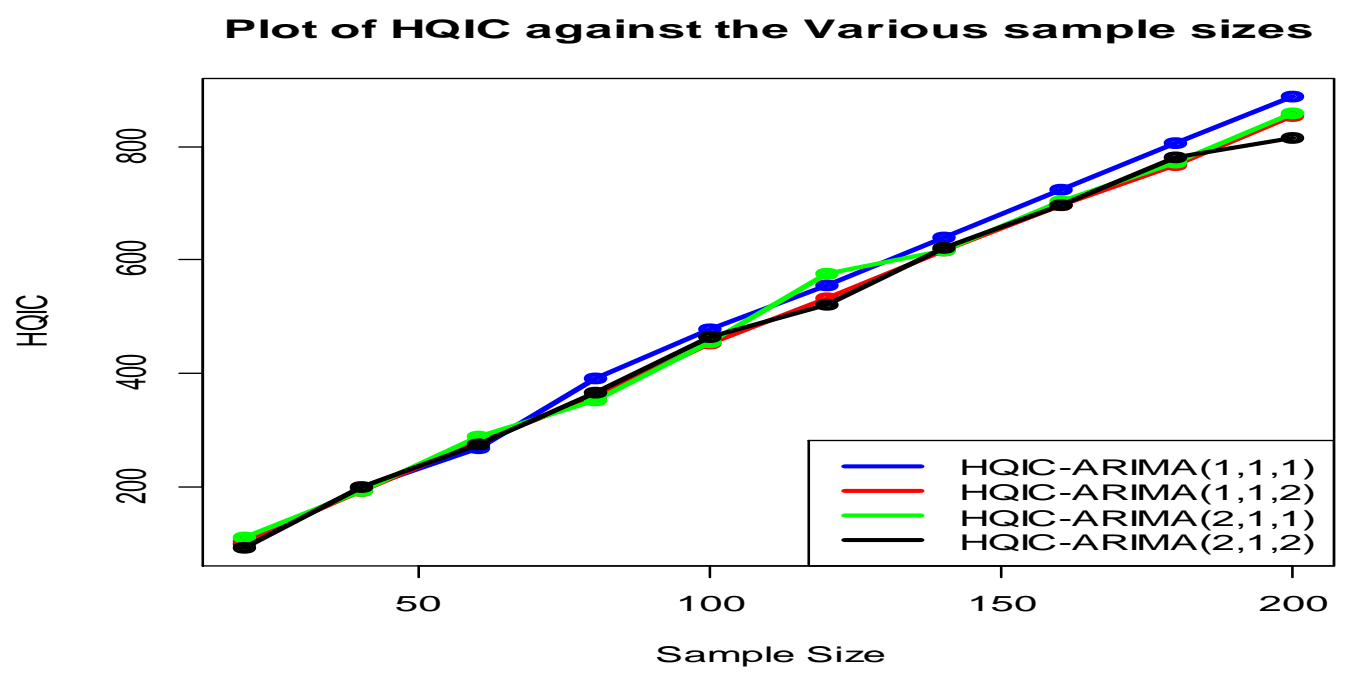

Fig.3.4a: HQIC values for ARIMA (p, d, q) Models from a Non-normal Data 


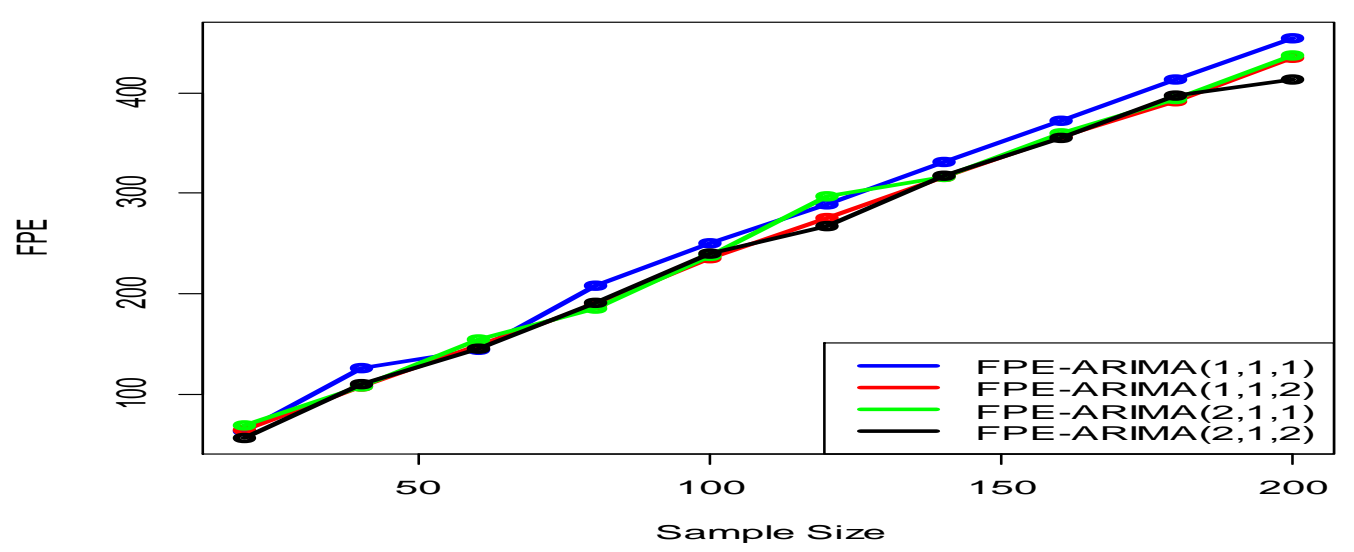

Fig.3.4b: FPE values for ARIMA (p, d, q) Models from a Non-normal Data

Similarly, table 3.4 above shows the relative performance of the four fitted models on the simulated data with 1000 iterations at various sample sizes. The average values of HQIC and FPE are recorded and then plotted in figures 3.4a and 3.4b. At sample sizes 20 , ARIMA $(1,1,1)$ was selected as the best fit. ARMA $(2,1,2)$ was the best fit at sample sizes $40,60,80,140,160,180$ and 200 respectively. While ARIMA $(1,1,2)$ was the best fit at 120

\section{0: Conclusion and Recommendation}

The general conclusion is that for Non-stationary Non-normal data, ARMA smaller orders were picked at almost all the sample sizes, for ARMA and ARIMA respectively. That at both lower $(20,40)$ and larger $(160,180$ and 200$)$ sample sizes, models with smallest orders [ARMA $(1,2)$ and ARMA $(2,1)]$ were picked on the average while at medium sample sizes, models with larger orders were picked. Similarly, for ARIMA (p, d, q) models, from sample sizes 0f 20-60, models with smaller orders were picked [ARIMA $(1,1,2)$ and ARIMA $(2,1,1)$ ] and at medium sample sizes from80-120 larger orders were picked ARIMA (2, $1,2)$. The selections are almost identical in both $d$ non-normal data structures, but vary with the variation in the distribution of the series.

Based on the results of the present study there is the need to develop a methodology for model selection combining objective and subjective techniques. Since, majority of researchers are unlikely to know the type of ARIMA process underlying the data understudy, so it to avoid relying on precarious procedures.

\section{REFERENCES}

Akaike, H., (1976). Canonical correlation analysis of Time Series and the use of an Information Criterion In Mehra, R.K., Lainiotis, D.G. (Eds.), Systems Identification: Advances and Case Studies. Academic Press, New York, pp. 27-96

Burnham K. P., Anderson D. R. and Huyvaert K. P. AIC model selection and multimodel inference in behavioral ecology: some background, observations, and comparisons [J]. Behav Ecol Sociobiol, 2011, 65:23-35

Chan, W. S. (1999). A comparison of some of Pattern Identification Methods for order Determination of Mixed ARMA models Statistics\& Probability Letters, $42,69-79$.

Chang, I. (1982) Outliers in Time Series; Ph.D Dissertation, Department of Statistics, University of Wisconsin, Madison, USA 
Cryer J. D. \& Chan K.S (2008) Time Series Analysis with Applications in R Second Edition, Springer Science Business Media, LLC

Davies, N., Petruccelli, J.D., (1984). On the use of the general partial autocorrelation function for order determination in ARMA (p; q) processes. J. Amer. Statist. Assoc. 79, 374\{377

$\begin{array}{lccccccccccc}\text { Eija } & \text { Ferreira.(2015) } & \text { Model } & \text { Selection } & \text { in } & \text { Time } & \text { Series } & \text { Machine } & \text { Learning } & \text { Applications } & \text { [D]. } \\ \text { Academic } & \text { dissertation } & \text { of } & \text { Technology } & \text { and } & \text { Natural } & \text { Sciences } & \text { of } & \text { the } & & \end{array}$

Hastie T., Tibshirani R. and Friedman J.( 2009) The Elements of Statistical Learning: Data Mining [J]. Inference and Prediction. Springer Series in Statistics. Springer-Verlag, New York, NY, 2nd edition,.

Martin, R.D. - Yohai, V.J. (1986) Influence Functional for Time Series (with discussion). The Annals of Statistics 14, 781-818.

Norhayati Y.( 2016) SURE-Autometrtcs Algorithm for Model Selection in Multiple Equations [D]. PhD Thesis, Universiti, Utara, Malaysia,

Ongbali S. O. , Igboanugo A. C., Afolalu S. A., Udo M. O. and Okokpujie I. P.(2018) Model Selection Process in Time Series Analysis of Production System with Random Output: Materials Science and Engineering Journal 